
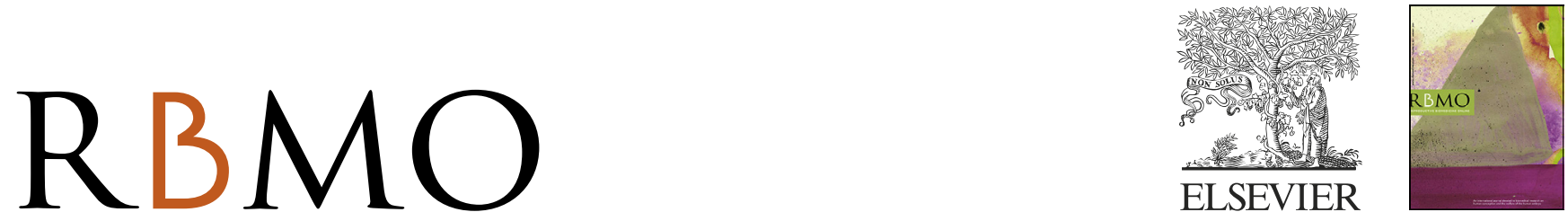

ARTICLE

\title{
The long-term costs and effects of tubal flushing with oil-based versus water-based contrast during hysterosalpingography
}

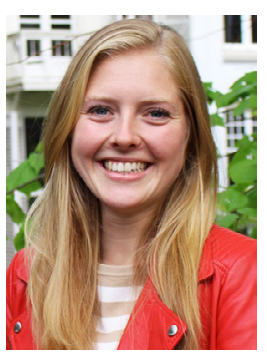

\author{
BIOGRAPHY \\ Nienke van Welie obtained her medical degree at the University of Amsterdam, the \\ Netherlands, in 2017. She is currently working as a PhD researcher at the Department of \\ Reproductive Medicine of Amsterdam University Medical Center. Her research project \\ focuses on diagnostic and therapeutic consequences of evaluation of the Fallopian tubes. \\ Nienke van Welie ${ }^{1, *}$, Clarabelle T. Pham ${ }^{2}$, Joukje van Rijswijk ${ }^{1}$, Kim Dreyer ${ }^{1}$, \\ Harold R. Verhoeve ${ }^{3}$, Annemieke Hoek ${ }^{4}$, Jan Peter de Bruin ${ }^{5}$, Annemiek \\ W. Nap ${ }^{6}$, Machiel H.A. van Hooff ${ }^{7}$ Mariëtte Goddijn ${ }^{8}$, Angelo B. Hooker', \\ Anna P. Gijsen ${ }^{10}$, Maaike A.F. Traas ${ }^{11}$, Jesper M.J. Smeenk ${ }^{12}$, Alexander \\ V. Sluijmer ${ }^{13}$, Marieke J. Lambers ${ }^{14}$, Gijsbertus A. van Unnik ${ }^{15}$, Cornelia \\ H. de Koning ${ }^{16}$, Alexander Mozes ${ }^{17}$, Catharina C.M. Timmerman ${ }^{18}$, Cornelis \\ B. Lambalk', Jonathan D. Karnon'2, Velja Mijatovic', Ben Willem J. Mol ${ }^{19,20}$ \\ on behalf of the H2Oil Study Group
}

\section{KEY MESSAGE}

Tubal flushing with oil-based contrast, compared with water-based contrast, had equivalent 5-year follow-up costs for a $7.5 \%$ increase in live birth rate. The increased price of oil-based contrast medium was compensated by a lower IVF uptake. The authors recommend it as the preferred strategy for tubal testing in infertile women with unexplained infertility.

\footnotetext{
1 Department of Reproductive Medicine, Amsterdam UMC, Amsterdam Reproduction and Development Research Institute, Vrije Universiteit Amsterdam Amsterdam, the Netherlands

${ }^{2}$ Flinders Health and Medical Research Institute, Flinders University, Adelaide, Australia

${ }^{3}$ Department of Obstetrics and Gynaecology, OLVG, Amsterdam, the Netherlands

${ }^{4}$ Department of Reproductive Medicine and Gynaecology, University of Groningen, University Medical Centre Groningen, Groningen, the Netherlands

${ }^{5}$ Department of Obstetrics and Gynaecology, Jeroen Bosch Hospital, 's Hertogenbosch, the Netherlands

${ }^{6}$ Department of Obstetrics and Gynaecology, Rijnstate Hospital, Arnhem, the Netherlands

7 Department of Obstetrics and Gynaecology, Franciscus Hospital, Rotterdam, the Netherlands

${ }^{8}$ Centre for Reproductive Medicine, Amsterdam UMC, University of Amsterdam, Amsterdam, the Netherlands

9 Department of Obstetrics and Gynaecology, Zaans Medical Centre, Zaandam, the Netherlands

${ }^{10}$ Department of Obstetrics and Gynaecology, Elkerliek Hospital, Helmond, the Netherlands

11 Department of Obstetrics and Gynaecology, Gelre Hospitals, Apeldoorn, the Netherlands

12 Department of Obstetrics and Gynaecology, Elisabeth-TweeSteden Hospital, Tilburg, the Netherlands

13 Department of Obstetrics and Gynaecology, Wilhelmina Hospital, Assen, the Netherlands

14 Department of Obstetrics and Gynaecology, Dijklander Hospital, Hoorn, the Netherlands

15 Department of Obstetrics and Gynaecology, Alrijne Hospital, Leiden, the Netherlands

${ }^{16}$ Department of Obstetrics and Gynaecology, Tergooi Hospital, Blaricum, the Netherlands

17 Department of Obstetrics and Gynaecology, Amstelland Hospital, Amstelveen, the Netherlands

${ }^{18}$ Department of Obstetrics and Gynaecology, Bravis Hospital, Roosendaal, the Netherlands

19 Department of Obstetrics and Gynaecology, Monash University, Clayton, Australia

${ }^{20}$ Aberdeen Centre for Women's Health Research, University of Aberdeen, Aberdeen, United Kingdom
}

(c) 2020 The Author(s). Published by Elsevier Ltd on behalf of Reproductive Healthcare Ltd. This is an open access article under the CC BY license (http://creativecommons.org/licenses/by/4.0/)

${ }^{*}$ Corresponding author. E-mail address: n.vanwelie@amsterdamumc.nl (N. van Welie). https://doi.org/10.1016/j. rbmo.2020.09.015 1472-6483/@ 2020 The Author(s). Published by Elsevier Ltd on behalf of Reproductive Healthcare Ltd. This is an open access article under the CC BY license (http://creativecommons.org/licenses/by/4.0/) Declaration of interest: C.T.P. has received consultancy fees for external work from Guerbet, France. K.D. reports receiving travel and speakers fee from Guerbet. H.R.V. reports receiving consultancy fees from Ferring. M.G. works at the Department of Reproductive Medicine of the Amsterdam UMC (location AMC and location VUmc). Location VUmc has received severa research and educational grants from Guerbet, Merck and Ferring. C.B.L. reports speakers fee from Ferring in the past, and his department receives research grants from Ferring, Merck and Guerbet. V.M. reports receiving travel and speakers fees as well as research grants from Guerbet. B.W.J.M. is supported by a NHMRC Investigator grant (GNT1176437). B.W.J.M. has received research grants from Merck and Guerbet. The other authors report no financial or commercial conflicts of interest.

\section{KEYWORDS}

Cost-Effectiveness

Female Infertility

Hysterosalpingography

Oil-based Contrast

Ongoing Pregnancy

Water-based Contrast 


\begin{abstract}
Research question: What are the long-term costs and effects of oil- versus water-based contrast in infertile women undergoing hysterosalpingography (HSG)?
\end{abstract}

Design: This economic evaluation of a long-term follow-up of a multicentre randomized controlled trial involved 1119 infertile women randomized to HSG with oil- $(n=557)$ or water-based contrast $(n=562)$ in the Netherlands.

Results: In the oil-based contrast group, 39.8\% of women needed no other treatment, $34.6 \%$ underwent intrauterine insemination (IUI) and $25.6 \%$ had IVF/intracytoplasmic sperm injection (ICSI) in the 5 years following HSG. In the waterbased contrast group, $35.0 \%$ of women had no other treatment, $34.2 \%$ had IUI and $30.8 \%$ had IVF/ICSI in the 5 years following HSG $(P=0.113)$. After 5 years of follow-up, HSG using oil-based contrast resulted in equivalent costs (mean cost difference -€144; 95\% confidence interval [Cl] -€579 to $+€ 290 ; P=0.515$ ) for a $5 \%$ increase in the cumulative ongoing pregnancy rate compared with HSG using water-based contrast (80\% compared with 75\%, Relative Risk (RR) 1.07; 95\% Cl 1.00-1.14). Similarly, HSG with oil-based contrast resulted in equivalent costs (mean cost difference -€50; $95 \% \mathrm{Cl}-€ 576$ to $+€ 475 ; P=0.850)$ for a $7.5 \%$ increase in the cumulative live birth rate compared with $\mathrm{HSG}$ with water-based contrast (74.8\% compared with 67.3\%, RR 1.11; 95\% Cl 1.03-1.20), making it the dominant strategy. Scenario analyses suggest that the oil-based contrast medium is the dominant strategy up to a price difference of $€ 300$.

Conclusion: Over a 5-year follow-up, HSG with an oil-based contrast was associated with a $5 \%$ increase in ongoing pregnancy rate, a $7.5 \%$ increase in live birth rate and similar costs to HSG with water-based contrast.

\section{INTRODUCTION}

\section{T}

raditionally, assessment of the Fallopian tubes is an important part of the fertility workup in infertile women.

Hysterosalpingography (HSG) is one of the most commonly applied outpatient clinic tubal patency tests in many countries and can be performed with either oil-based or water-based contrast medium (Cundiff et al., 1995). HSG was initially introduced as a diagnostic test, but possible therapeutic effects of tubal flushing have been suggested in literature for many years, especially with the use of oil-based contrast (Mohiyiddeen et al., 2015; Watson et al., 1994; Weir and Weir, 1951,).

In 2017, a large randomized controlled trial (RCT) in the Netherlands, the H2Oil study, confirmed that HSG with oil-based contrast (Lipiodol Ultra-Fluid; Guerbet, France) resulted in 10\% higher 6-month ongoing pregnancy and live birth rates compared with HSG with water-based contrast (Telebrix Hystero; Guerbet, France) (Dreyer et al., 2017). A costeffectiveness analysis of the H2Oil study showed that HSG using an oil-based contrast was a cost-effective strategy after a 6-month (short-term) follow-up, with an incremental cost of US $\$ 8198$ for an additional ongoing pregnancy compared with HSG using a water-based contrast (van Rijswijk et al., 2018).

Considering these results, tubal flushing with oil-based contrast is gaining popularity, and two meta-analyses have recently been published on this topic (Fang et al., 2018; Wang et al., 2019).
However, the follow-up duration of the included studies varies widely, and none of the included studies addressed the possible long-term effects of tubal flushing. Evidence regarding long-term fertility-enhancing effects of tubal flushing was insufficient until recently. In 2020 van Rijswijk and colleagues published the H2Oil long-term follow-up study, comparing the effects of oil-versus water-based contrast medium at HSG up to 5 years after randomization in the H2Oil study (van Rijswijk et al., 2020). This follow-up study showed a higher 5-year cumulative ongoing pregnancy rate, a shorter time to pregnancy and a higher chance of a natural conception in favour of oil-based contrast up to 5 years after randomization. However, the long-term costs and effects of oil-based contrast have not yet been studied. The control of costs is important to facilitate access to effective fertility care. In view of increasing healthcare costs (Organisation for Economic Co-operation and Development, 2017), it is important to investigate and compare the long-term costs and effects of the two different types of contrast medium. The aim of this study is to assess the long-term costs and effects of HSG with oil-based contrast versus water-based contrast in a long-term cost-effectiveness analysis.

\section{MATERIALS AND METHODS}

This study involved an economic evaluation of the long-term followup of the $\mathrm{H} 2 \mathrm{Oil}$ study (Netherlands Trial Register (NTR) 6577, http://www. trialregister.nl) of participants in the H2Oil study (NTR 3270). Reproductive outcome data up to 5 years after randomization were collected. Details of the H2Oil study and H2Oil follow-up study have been published elsewhere (Dreyer et al., 2017; van Rijswijk et al., 2020). This study was investigator initiated, and the research protocol was approved by the Institutional Review Board of the Amsterdam University Medical Centre - VU University Medical Centre (reference 2017.221, dated 14 June 2017). In this article, the trial essentials are briefly discussed.

Patients and study procedures Infertile women between 18 and 39 years of age with an ovulatory cycle and an indication for tubal patency testing by $\mathrm{HSG}$ were eligible for the $\mathrm{H} 2 \mathrm{Oil}$ study. Couples with male infertility (total motile sperm count after sperm washing of less than 3 million spermatozoa per millilitre), endocrine disorders (e.g. polycystic ovary syndrome, diabetes, hyperthyroidism or hyperprolactinaemia), iodine allergy or a high risk of tubal pathology (a history of pelvic inflammatory disease, previous Chlamydia infection or known endometriosis) were excluded. After informed consent, women were randomized to the use of oil-based contrast (Lipiodol Ultra Fluid; Guerbet, France) or water-based contrast (Telebrix Hystero; Guerbet, France) during HSG in a 1:1 ratio, and HSG was performed according to local protocols (Dreyer et al., 2017; van Rijswijk et al., 2020).

Infertility treatments Fertility treatment advice within the 12 months after HSG was based on the standard Hunault prediction for natural 


\section{TABLE 1 UNIT COSTS FOR THE CONTRAST MEDIUM INTERVENTION, INFERTILITY TREATMENTS, AND PREGNANCY AND DELIVERY OR MISCARRIAGE (€)}

\begin{tabular}{lll}
\hline Characteristics & Unit costs (€) & Source \\
\hline Contrast medium & 225 & Guerbet, France \\
\hline Oil-based contrast & 12 & Guerbet, France \\
\hline Water-based contrast & & \\
\hline Infertility treatments & 300 & Tjon-Kon-Fat et al. (2015) \\
\hline Intrauterine insemination & 3,000 & Tjon-Kon-Fat et al. (2015) \\
\hline IVF & 3,300 & Tjon-Kon-Fat et al. (2015) \\
\hline Intracytoplasmic sperm injection & & Lukassen et al. (2004) \\
\hline Pregnancy and delivery & 3,320 & Lukassen et al. (2004) \\
\hline Singleton & 17,540 & Graziosi et al. (2005)
\end{tabular}

conception (Hunault et al., 2005).

This prognostic model is widely used

in the Netherlands as a decision aid.

Depending on the results of the HSG,

women received expectant management

for 6 months if their prognosis for

natural conception was good ( $>30 \%$ in

12 months), or underwent intrauterine insemination (IUI) if their prognosis for natural conception was less than $30 \%$ or 6 months of expectant management had elapsed (Hunault et al., 2005). Women underwent IVF/intracytoplasmic sperm injection (ICSI) if there was bilateral tubal occlusion, semen analysis during IUI showed very poor semen quality or there had been six failed IUI cycles (van Rijswijk et al., 2020). The main outcome was first ongoing pregnancy after HSG, defined as a positive fetal heartbeat on ultrasound after 12 weeks of gestation. Secondary outcomes were live birth, defined as a live birth after 24 weeks of gestation, multiple pregnancy rate and miscarriage rate. Additional secondary outcomes measured in the H2Oil study were not considered in this economic evaluation.

\section{Economic evaluation}

The economic evaluation was performed from the healthcare system perspective. The analysis was conducted according to the International Society for Pharmacoeconomics and Outcomes Research (ISPOR) guidelines (McGhan et al., 2009), and the report followed the Consolidated Health Economic Evaluation Reporting Standards statement (Husereau et al., 2013).

For the primary outcome of first ongoing pregnancy leading to live birth, the total direct medical costs for each participant included those for the contrast medium intervention and infertility treatments and miscarriage in the 5 years since randomization. An additional analysis included the direct medical costs related to pregnancy and delivery to assess the costs associated with achieving a live birth.

Data on resource use were obtained by reviewing each participant's electronic medical records; in addition, a questionnaire was sent to all $\mathrm{H} 2 \mathrm{Oil}$ participants to collect information supplementary to their medical record (van Rijswijk et al., 2020). For each participant, the type and number of interventions received either until the first ongoing pregnancy or within 5 years since randomization were registered, as was whether a single or multiple (twin) live births were achieved. The costs for each parameter were derived from three previous publications (Graziosi et al., 2005; Lukassen et al., 2004; Tjon-Kon-Fat et al., 2015). The costs for the contrast media were provided by the manufacturer (Guerbet, France). The cost parameters and unit costs included in the economic evaluation for the follow-up study (expressed in euros) are presented in TABLE 1. All calculations were standardized to 2018 prices using consumer price index data. Three main cost categories were used: contrast media, infertility treatments, and pregnancy and delivery or miscarriage.

\section{Statistical analysis}

The mean costs and outcomes for each treatment group were compared.
Incremental cost-effectiveness ratios and $95 \%$ confidence intervals $(\mathrm{Cl})$ were calculated by dividing the difference in total costs by the difference in outcome between the two groups. Bootstrapping (using 1000 resamples) was used to represent the joint uncertainty around the incremental costs and outcomes. A cost-effectiveness plane was generated to graphically represent the difference in costs and outcomes between oil-based and water-based contrast media and the uncertainty around the expected costs and expected effects associated with each intervention. Cost-effectiveness acceptability curves were also generated to indicate the probability that use of the oil-based contrast medium would be cost-effective compared with waterbased contrast medium for a range of values representing the willingness to pay for ongoing pregnancy and live birth. Statistical analyses were performed using Stata Statistical Software, version 15 (StataCorp, USA) and Microsoft Excel 2016 (Microsoft Corporation, USA). Data were analysed using the intention to treat principle.

\section{Scenario analyses}

As a comparison, scenario analyses were undertaken to determine the impact of the difference in contrast media cost on the cost-effectiveness. The base case difference in the costs of the contrast media was $€ 213$, values ranging from $€ 0$ to $€ 500$ were tested.

\section{RESULTS}

\section{Study population}

Between February 2012 and October 2014, 1119 women were randomly assigned to HSG with oil-based contrast ( $n=557$ ) or water-based contrast $(n=562)$. The baseline characteristics were similar across the two groups (Supplementary Table S1). Five women were lost to follow-up within the first 6 months: two from the oil group and three from the water group. Due to the low number of missing values, missing data were not imputed. Thus, the analyses were undertaken on 555 women in the oil group and 559 women in the water group (1114 in total).

Participants' characteristics and trial follow-up outcomes, including infertility treatments after HSG, are summarized in Supplementary Table S1. Of the women in the oil-based contrast medium group, $39.8 \%$ had no other treatment, 
TABLE 2 COMPARISON OF MEAN COSTS PER WOMAN FOR THE TWO TREATMENT GROUPS IN EUROS

\begin{tabular}{lll}
\hline Cost parameter & Oil group $(\boldsymbol{n}=\mathbf{5 5 5})(\boldsymbol{\epsilon})$ & Water group (n= 559) (€) \\
\hline Contrast medium & 225 & 12 \\
\hline Infertility treatments & $2102(3539)$ & $2459(3848)$ \\
\hline Total costs (for ongoing pregnancy) & $2327(3539)$ & $2471(3848)$ \\
\hline Miscarriage & $212(568)$ & $254(613)$ \\
\hline Pregnancy and delivery & $2713(2388)$ & $2577(2859)$ \\
\hline Total costs (for live birth) & $5252(4062)$ & $-357(-792$ to 77$), P=0.107$ \\
\hline
\end{tabular}

Data are presented as mean and standard deviation, unless otherwise specified.

$\mathrm{Cl}$, confidence interval.

$34.6 \%$ underwent IUI and $25.6 \%$ had IUI followed by IVF/ICSI or IVF/ICSI alone in the 5 years following HSG. In comparison, of the women in the waterbased contrast medium group, 35.0\% had no other treatment, $34.2 \%$ had IUI and $30.8 \%$ had IUI followed by IVF/ICSI or IVF/ICSI alone in the 5 years following HSG $(P=0.113)$. The ongoing pregnancy rates within the 5 years were $80 \%$ in the oil-based contrast group and $75 \%$ in the water-based contrast group (Relative Risk (RR) 1.07; 95\% Cl 1.00-1.14). The live birth rates within the 5 years were $75 \%$ in the oil-based contrast group and $67 \%$ in the water-based contrast group (RR 1.11; 95\% Cl 1.03-1.20). Information on live birth was missing in 20 ongoing pregnancies in the oil group versus 33 ongoing pregnancies in the water group. The multiple pregnancy and miscarriage rates within the 5 years were low and did not differ significantly. Median followup was 45.3 months in the oil-based contrast group and 46.7 months in the water-based contrast group.

\section{Costs and effects (oil-based contrast versus water-based contrast) \\ After 5 years of follow-up, the difference in the proportion of women with an ongoing pregnancy was $5.0 \%(95 \% \mathrm{Cl}$ $0.03-9.9 \% ; 80.0 \%$ in the oil group versus $75.0 \%$ in the water group) and with a live birth was $7.5 \%$ (95\% Cl 2.1-12.5\%; $74.8 \%$ in the oil group versus $67.3 \%$ in the water group).}

A comparison of the mean costs per woman by cost category for the two treatment groups is provided in TABLE 2. The mean costs per woman were lower in the oil-based contrast group than in the water-based contrast group for ongoing pregnancy (mean cost difference $-€ 144 ; 95 \% \mathrm{Cl}-€ 579$ to $+€ 290$; $P=0.515)$. For the oil-based group, the higher cost for the contrast medium
(€225) was offset by more women requiring no other treatment (i.e. 39.8\% conceived naturally) and fewer women having IVF/ICSI (25.6\%). The water-based group had a lower contrast medium cost (€12) but more women had IVF/ICSI $(30.8 \%)$ and fewer women conceived naturally (35.0\%). The proportion of women having IUI was similar across the two treatment groups.

Similarly, for live birth, the mean costs per woman were lower in the oil-based contrast group than in the water-based contrast group (mean cost difference $-€ 50 ; 95 \% \mathrm{Cl}-€ 576$ to $+€ 475$;

$P=0.850)$. The smaller difference in mean costs was due to the increased costs for pregnancy and delivery as a result of the increase in live births in the oil-based group. Thus, after 5 years of follow-up, HSG using an oil-based contrast medium was considered the dominant strategy over HSG using a water-based contrast medium, with comparable costs and higher ongoing pregnancy and live birth rates.

The bootstrap samples were located in the eastern quadrants, indicating that there is little uncertainty regarding the effectiveness of the oil-based contrast medium (FIGURE 1). The spread of the bootstrap samples across the north and south-eastern quadrants indicates that there is uncertainty regarding the cost difference between women receiving water- and oil-based contrast media (FIGURE 1). Given the cost-effectiveness acceptability curves presented in FIGURE 2, if the maximum acceptable ratio is $€ 4000$ per additional ongoing pregnancy or live birth, the probability that the use of an oil-based contrast medium is cost-effective compared with a water-based contrast medium is 0.9 . Alternatively, this can be interpreted as there being a $90 \%$ chance that the potential additional cost of an oil-based contrast medium, compared with a water-based contrast medium, is less than $€ 4000$ per additional ongoing pregnancy or live birth. The costs associated with pregnancy and delivery had the greatest impact on the costeffectiveness of the oil-based contrast medium compared with the water-based contrast medium (TABLE 2).

\section{Scenario analyses}

The impact of the difference in contrast media costs on cost-effectiveness is presented in Supplementary Table S2. If there is no difference in the cost of contrast medium, the oil-based contrast medium is the dominant strategy for both ongoing pregnancy and live birth until a cost difference of $€ 300$, at which the oil-based contrast medium is no longer less costly and more effective, with a mean incremental cost-effectiveness ratio of $€ 493$ for an additional live birth.

\section{DISCUSSION}

An economic evaluation was performed alongside a long-term follow-up study of an RCT including infertile women scheduled for HSG with oil-based versus water-based contrast medium during a fertility workup. Women allocated to HSG with oil-based contrast medium had higher ongoing pregnancy and live birth rates than women allocated to HSG with water-based contrast for equivalent costs. After 5 years of follow-up, HSG with oil-based contrast resulted in equivalent costs (mean cost difference -€144; 95\% $\mathrm{Cl}-€ 579$ to $+€ 290 ; \mathrm{P}=0.515$ ) for a $5 \%$ higher cumulative ongoing pregnancy rate. Similarly, HSG with oil-based contrast lead to equivalent costs (mean cost difference -€50; 95\% Cl -€576 to $+€ 475 ; P=0.850$ ) for a $7.5 \%$ higher cumulative live birth rate compared with HSG with water-based contrast. These 


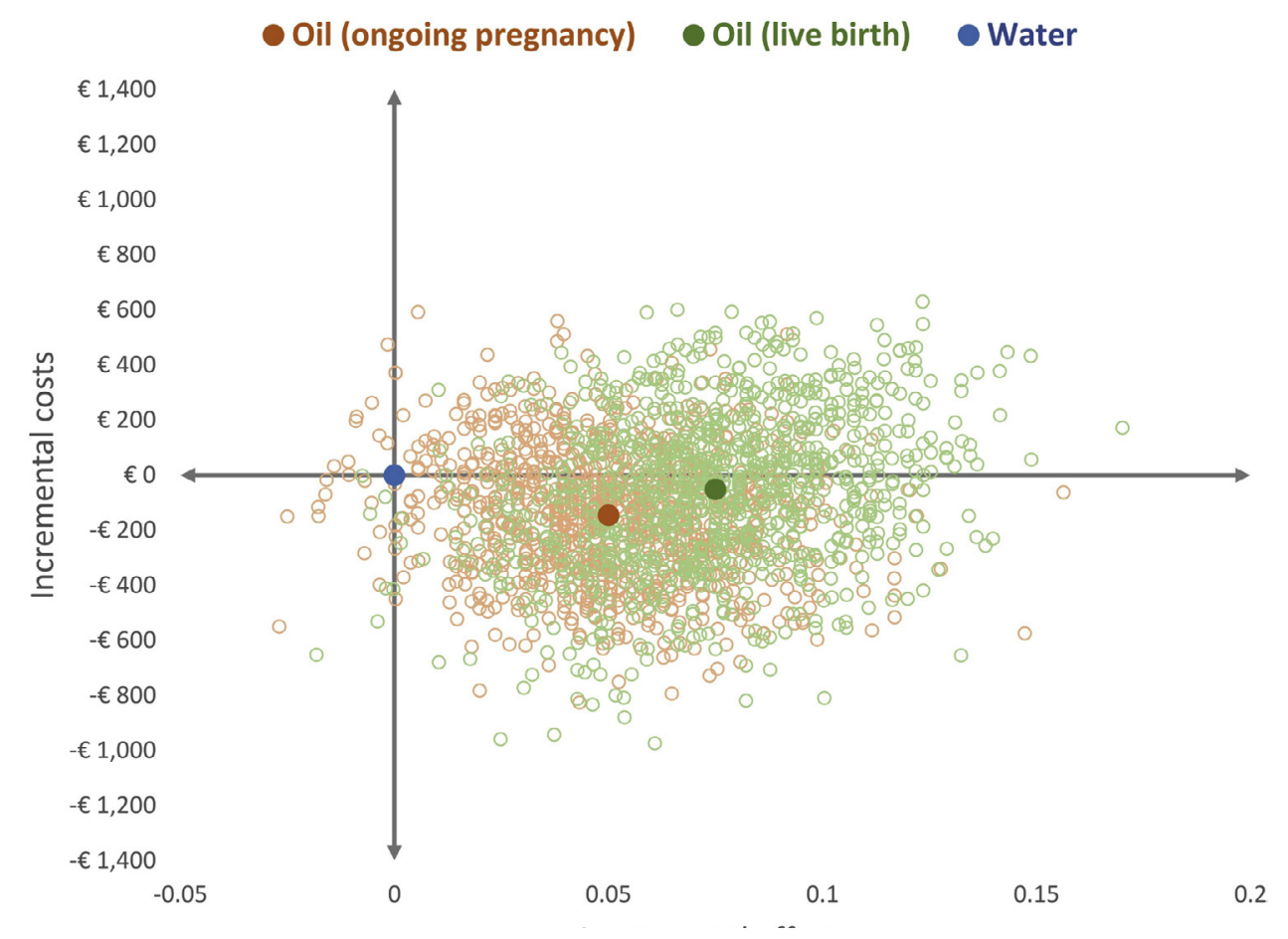

FIGURE 1 The cost-effectiveness plane representing the costs and effects of the oil-based contrast medium relative to the water-based contrast medium and the uncertainty around the incremental cost-effectiveness ratios. Estimates in the north-western quadrant of the cost-effectiveness plane indicate that the intervention is less effective and more costly; estimates in the south-western quadrant indicate that it is less effective and less costly; estimates in the south-eastern quadrant indicate that is is more effective and less costly; and estimates in the north-eastern quadrant indicate that it is more effective and more costly. Closed circles represent the base case incremental cost-effectiveness ratio (ICER) estimate; open circles represent the uncertainty around each ICER.

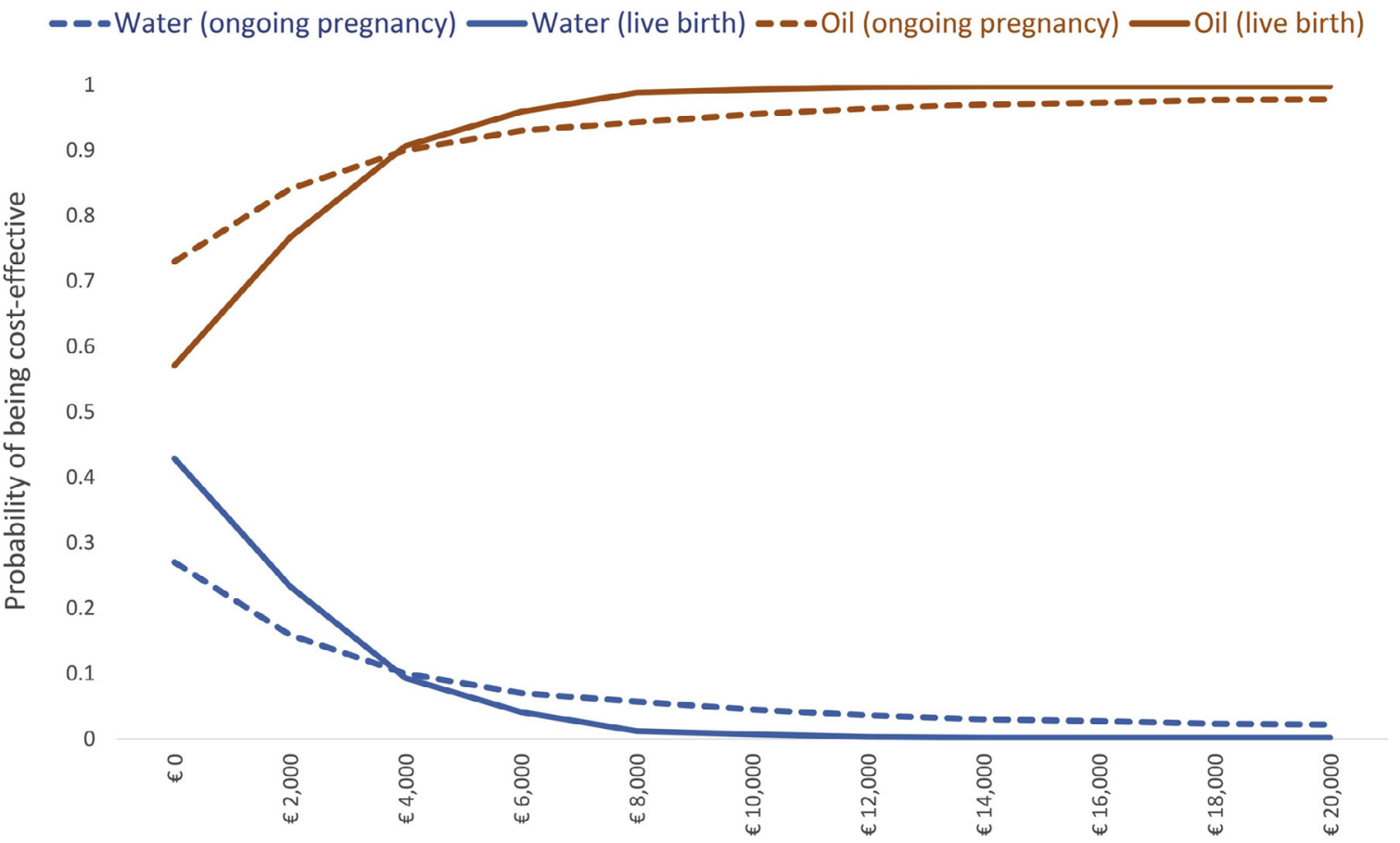

FIGURE 2 Cost-effective acceptability curves for ongoing pregnancy and live birth, indicating the probability that using oil-based contrast medium is cost-effective regarding ongoing pregnancy (dotted lines) and live birth (solid lines) after 3-5 years of follow-up, given alternative monetary values. 
differences make HSG with oil-based contrast the dominant strategy in infertile women with unexplained or mild male factor infertility.

This study has several strengths, but also some limitations. Its main strength is that this follow-up study was based on a large multicentre RCT investigating the effects of oil-based versus waterbased contrast with prospective and retrospective registration of resource use. The lost to follow-up at 6 months was lower in this follow-up study (two in the oil group versus three in the water group) than in the original $\mathrm{H} 2 \mathrm{Oil}$ trial (five in the oil group versus 11 in the water group), so more data were available combining information from the medical files and the returned questionnaires. Furthermore, studies regarding the long-term costs and effects of oil- versus water-based contrast were lacking, and this study contributes to the counselling of couples undergoing tubal patency testing during fertility workup as well as giving guidance to healthcare policy makers.

A limitation of the study is that data regarding the long-term follow-up were collected retrospectively by reviewing medical files and sending women a questionnaire, resulting in variable durations of follow-up. However, the median time to follow-up was comparable between the two groups, which allowed a comparison between absolute ongoing pregnancy and live birth rates. Furthermore, information on live birth was missing in 20 ongoing pregnancies in the oil group versus 33 ongoing pregnancies in the water group, either because there was no response on the questionnaire or because the woman was still pregnant at time of data collection. If it is assumed that all who were missing had a live birth, the difference in live birth between the oil and water groups would be 5\% (the same as the difference in ongoing pregnancy), and the costs for pregnancy and delivery would increase accordingly.

Second, this economic evaluation focused on the direct medical costs and did not include potential indirect costs for patients and society (e.g. loss of productivity during pregnancy and delivery) as these data were not collected during the trial follow-up. It might be expected that, over a 3- to 5 -year period, the cost difference could increase in the case of more ongoing pregnancies and subsequently more hospital visits, transportation costs and productivity loss. On the other hand, visiting a fertility clinic and undergoing assisted reproductive technology (ART) also increases transportation costs and productivity loss.

Third, as this study was executed in the Netherlands, Netherlands-based costs were used. The price difference between oil-based and water-based contrast media was $€ 213$ ( $€ 225$ versus $€ 12$ ). Globally, however, the cost differences of the two contrast media vary widely, thus limiting the generalizability of the data. Therefore, scenario analyses were performed illustrating the impact of the difference in contrast medium cost on cost-effectiveness, to make the results data applicable to other countries. HSG with oil-based contrast is cost-effective with a price difference of $€ 300$ or lower. However, in countries with high price differences, HSG with oil-based contrast is less cost-effective, and implementation depends on what society is willing to pay for an additional child.

Fourth, this study was limited to women at low risk of tubal pathology, less than 39 years of age and with no known endocrinological diseases. The findings should therefore not be generalized to other groups of infertile women.

A comparison of the results can be made with those of other studies. At 6 months, HSG using oil-based contrast is a costeffective strategy compared with the use of water-based contrast, if society is willing to pay US \$8198 per extra ongoing pregnancy (van Rijswijk et al., 2018). The cost difference between the two types of contrast media is globally the highest in the USA (US\$900), and ART costs in the USA are much higher than those in Europe. HSG using oil-based contrast leads to more natural and IUI-conceived pregnancies and fewer IVF or ICSIconceived pregnancies in the long term. The cost-effectiveness of this strategy would be largely influenced by the costs of the oil-based contrast medium and the healthcare costs associated with pregnancy and delivery.

Two studies have evaluated the costeffectiveness of tubal patency testing during fertility workup (Mol et al., 2001; Verhoeve et al., 2013). Both studies evaluated different scenarios for the live birth rate within 3 years after fertility workup, i.e. expectant management without diagnostics, immediate IVF without diagnostics, delayed IVF without diagnostics, HSG and IVF, laparoscopy and IVF, etc. Mol and colleagues found Chlamydia antibody testing during fertility workup to be less costly compared with HSG in women with a good fertility prospects, whereas immediate HSG was advised in women with relatively poor fertility prospects (Mol et al., 2001). Verhoeve and coworkers found that not performing a diagnostic tubal patency test but delaying IVF was the most cost-effective strategy in women younger than 38 years of age. In women above 38 years of age, no diagnostic tubal patency test and immediate IVF was the most cost-effective strategy (Verhoeve et al., 2013). However, these cost-effectiveness analyses did not take the type of contrast medium into account or the fertilityenhancing effect of oil-based contrast. Nevertheless, recent studies have shown a fertility-enhancing effect of tubal flushing itself independent of the type of contrast medium. The effect was more prominent with an oil-based contrast medium (Dreyer et al., 2019; Wang et al., 2019).

Implications for treatment can also be seen from the results of the current study. After 5 years of follow-up, HSG with oil-based contrast resulted in higher cumulative live birth rates compared with HSG with water-based contrast at an equivalent cost. The higher price of oil-based contrast was compensated by lower IVF or ICSI uptake in the oil group. More couples conceived naturally or by IUI after HSG with oil-based contrast, and fewer couples conceived by IVF or ICSI after HSG with oil-based contrast. This therefore suggests that offering women a tubal patency test using oilbased contrast during fertility workup is a good first step before starting ART, especially in countries where ART is not reimbursed. The presumed higher chance of an ongoing pregnancy after HSG could prevent couples from the burdens of ART. Additionally, HSG is a relatively easy procedure and is less timeconsuming. This study's findings can thus be used for counselling infertile couples during fertility workup.

In conclusion, over a 5-year follow-up period, HSG with oil-based contrast had similar costs for a $5 \%$ increase in 
the ongoing pregnancy rate and a 7.5\% increase in the live birth rate when compared with HSG with a water-based contrast.

\section{ACKNOWLEDGEMENTS}

The authors would like to thank all the participating women, the hospitals and their staff, the research nurses and the staff of the Nationwide Consortium for Women's Health Research (NVOG Consortium; www. zorgevaluatienederland. nl) for logistical support. Thanks also go to the $\mathrm{H} 2 \mathrm{Oil}$ study group collaborators: Nan van Geloven, Jos W. R. Twisk, Peter M. van de Ven and Peter G. A. Hompes for their contributions to this study. The original $\mathrm{H} 2 \mathrm{O}$ il RCT was an investigator-initiated study that was funded by the two academic institutions (AMC and VUmc) of the Amsterdam UMC. The long-term follow-up study and economic analysis, both investigator-initiated studies, were funded by a research grant from Guerbet, France. The funders had no role in study design or collection, analysis or interpretation of the data.

\section{SUPPLEMENTARY MATERIALS}

Supplementary material associated with this article can be found, in the online version, at doi:10.1016/j. rbmo.2020.09.015.

\section{APPENDIX: H2OIL STUDY GROUP MEMBERS}

Petra Bourdrez', Angelique J. C. M. van Dongen², Annette E. J. Duijn ${ }^{3}$, Ron J. T. van Golde ${ }^{4}$, Cathelijne F. van Heteren ${ }^{5}$, Mesrure Kaplan', Frederique D. Lichtenbeld7, Marie J. Pelinck ${ }^{8}$, Henrike G. M. van Rijnsaardt-Lukassen", Ilse A. J. van Rooij ${ }^{10}$ and Rachel Tros. ${ }^{11}$

1Department of Obstetrics and Gynaecology, VieCuri Medical Centre, Venlo, the Netherlands.

${ }^{2}$ Department of Obstetrics and Gynaecology, Hospital Gelderse Vallei, Ede, the Netherlands.

${ }^{3}$ Vrouwenkliniek Zuidoost, Amsterdam, the Netherlands.

${ }^{4}$ Department of Obstetrics and Gynaecology, Maastricht UMC, Maastricht, the Netherlands.
${ }^{5}$ Department of Obstetrics and Gynaecology, Canisius Wilhelmina

Hospital, Nijmegen, the Netherlands.

${ }^{6}$ Department of Obstetrics and Gynaecology, Röpcke-Zweers Hospital, Hardenberg, the Netherlands.

${ }^{7}$ Department of Reproductive Medicine, Amsterdam UMC, Vrije Universiteit Amsterdam, Amsterdam, the Netherlands.

${ }^{8}$ Department of Obstetrics and Gynaecology, Scheper Hospital, Emmen, the Netherlands.

${ }^{9}$ Department of Obstetrics and Gynaecology, Albert Schweitzer Hospital, Dordrecht, the Netherlands.

${ }^{10}$ Department of Obstetrics and Gynaecology, Elisabeth-TweeSteden Hospital, Tilburg, the Netherlands.

${ }^{11}$ Department of Obstetrics and Gynaecology, Amsterdam UMC, Vrije Universiteit Amsterdam, Amsterdam, the Netherlands.

\section{REFERENCES}

Cundiff, G, Carr, BR, Marshburn, PB Infertile couples with a normal hysterosalpingogram. Reproductive outcome and its relationship to clinical and laparoscopic findings. The Journal of reproductive medicine 1995; 40: 19-24

Dreyer, K, van Rijswijk, J, Mijatovic, V, Goddijn, M, Verhoeve, HR, van Rooij, IAJ, Hoek, A, Bourdrez, P, Nap, AW, Rijnsaardt-Lukassen, HGM, Timmerman, CCM, Kaplan, M, Hooker $A B$, Gijsen, AP, van Golde, R, van Heteren, CF, Sluijmer, AV, de Bruin, JP, Smeenk, JMJ, de Boer, JAM, Scheenjes, E, Duijn, AEJ, Mozes, A, Pelinck, MJ, Traas, MAF, van Hooff, $\mathrm{MHA}$, van Unnik, GA, de Koning, $\mathrm{CH}$, van Geloven, N, Twisk, JWR, Hompes, PGA, Mol, BWJ Oil-Based or Water-Based Contrast for Hysterosalpingography in Infertile Women. N Engl. J. Med. 2017; 376: 2043-2052

Dreyer, K, van Eekelen, R, Tjon-Kon-Fat, RI, van der Steeg, JW, Steures, P, Eijkemans, M, van der Veen, F, Hompes, P, Mol, B, van Geloven, N The therapeutic effect of hysterosalpingography in couples with unexplained subfertility: a post-hoc analysis of a prospective multi-centre cohort study. Reprod. Biomed. Online 2019; 38: 233-239

Fang, F, Bai, Y, Zhang, Y, Faramand, A Oilbased versus water-based contrast for hysterosalpingography in infertile women: a systematic review and meta-analysis of randomized controlled trials. Fertil. Steril. 2018; 110: 153-160

Graziosi, GC, van der Steeg, JW, Reuwer, PH, Drogtrop, AP, Bruinse, HW, Mol, BW Economic evaluation of misoprostol in the treatment of early pregnancy failure compared to curettage after an expectant management. Hum. Reprod. 2005; 20: 1067-1071

Hunault, CC, Laven, JS, van Rooij, IA, Eijkemans, MJ, te Velde, ER, Habbema, JD Prospective validation of two models predicting pregnancy leading to live birth among untreated subfertile couples. Hum. Reprod. 2005; 20: 1636-1641

Husereau, D, Drummond, M, Petrou, S, Carswell, C, Moher, D, Greenberg, D, Augustovski, F, Briggs, AH, Mauskopf, J, Loder, E Consolidated Health Economic Evaluation Reporting Standards (CHEERS)-explanation and elaboration: a report of the ISPOR Health Economic Evaluation Publication Guidelines Good Reporting Practices Task Force. Value in health: the journal of the International Society for Pharmacoeconomics and Outcomes Research 2013; 16: 231-250

Lukassen, HG, Schonbeck, Y, Adang, EM, Braat, DD, Zielhuis, GA, Kremer, JA Cost analysis of singleton versus twin pregnancies after in vitro fertilization. Fertil. Steril. 2004; 81: 1240-1246

McGhan, WF, Al, M, Doshi, JA, Kamae, I, Marx, SE, Rindress, D The ISPOR Good Practices for Quality Improvement of Cost-Effectiveness Research Task Force Report. Value in health: the journal of the International Society for Pharmacoeconomics and Outcomes Research 2009; 12: 1086-1099

Mohiyiddeen, L, Hardiman, A, Fitzgerald, C, Hughes, E, Mol, BW, Johnson, N, Watson, A Tubal flushing for subfertility. The Cochrane database of systematic reviews $2015 \mathrm{Cd} 003718$

Mol, BW, Collins, JA, Van Der Veen, F, Bossuyt, PM Cost-effectiveness of 
hysterosalpingography, laparoscopy, and Chlamydia antibody testing in subfertile couples. Fertil. Steril. 2001; 75: 571-580

Organisation for Economic Co-operation and Development. OECD Health Statistics. 2017.

Tjon-Kon-Fat, RI, Bensdorp, AJ, Bossuyt, PM, Koks, C, Oosterhuis, GJ, Hoek, A, Hompes,

P, Broekmans, FJ, Verhoeve, HR, de Bruin,

JP, van Golde, R, Repping, S, Cohlen, BJ,

Lambers, MD, van Bommel, PF, Slappendel, E,

Perquin, D, Smeenk, J, Pelinck, MJ, Gianotten,

J, Hoozemans, DA, Maas, JW, Groen, $H_{\text {, }}$

Eijkemans, MJ, van der Veen, F, Mol, BW, van

Wely, M Is IVF-served two different ways-more cost-effective than IUI with controlled ovarian hyperstimulation? Hum. Reprod. 2015; 30 2331-2339

van Rijswijk, J, Pham, CT, Dreyer, K, Verhoeve, HR, Hoek, A, de Bruin, JP, Nap, AW, Wang R, Lambalk, CB, Hompes, PGA, Mijatovic, V,
Karnon, JD, Mol, BW Oil-based or waterbased contrast for hysterosalpingography in infertile women: a cost-effective analysis of a randomized controlled trial. Fertil. Steril. 2018; 110: 754-760

van Rijswijk, J, Van Welie, N, Dreyer, K, Pham, CT, Verhoeve, HR, Hoek, A, De Bruin, JP, Nap, AW, van Hooff, MHA, Goddijn, M, Hooker, AB, Bourdrez, P, Van Dongen, AJCM, van Rooij, IAJ, Rijnsaardt-Lukassen, HGM, van Golde, RJT, Van Heteren, CF, Pelinck, MJ, Duijn, AEJ, Kaplan, M, Lambalk, CB, Mijatovic, V, Mol, BWJ Tubal flushing with oil- or water-based contrast at hysterosalpingography for infertility: long term reproductive outcomes of a randomized trial. Fertil. Steril. 2020

Verhoeve, HR, Moolenaar, LM, Hompes, P, van der Veen, F, Mol, BW Cost-effectiveness of tubal patency tests. BJOG: an international journal of obstetrics and gynaecology 2013; 120: 583-593

Wang, R, van Welie, N, van Rijswijk, J, Johnson, NP, Norman, RJ, Dreyer, K, Mijatovic, V, Mol, BW Effectiveness on fertility outcome of tubal flushing with different contrast media: systematic review and network meta-analysis. Ultrasound Obstet. Gynecol. 2019; 54: 172-181

Watson, A, Vandekerckhove, P, Lilford, R, Vail, A, Brosens, I, Hughes, E A meta-analysis of the therapeutic role of oil soluble contrast media at hysterosalpingography: a surprising result? Fertil. Steril. 1994; 61: 470-477

Weir, WC, Weir, DR. Therapeutic value of salpingograms in infertility. Fertil. Steril. 1951; 2: $514-522$

Received 5 March 2020; received in revised form 18 August 2020; accepted 11 September 2020. 